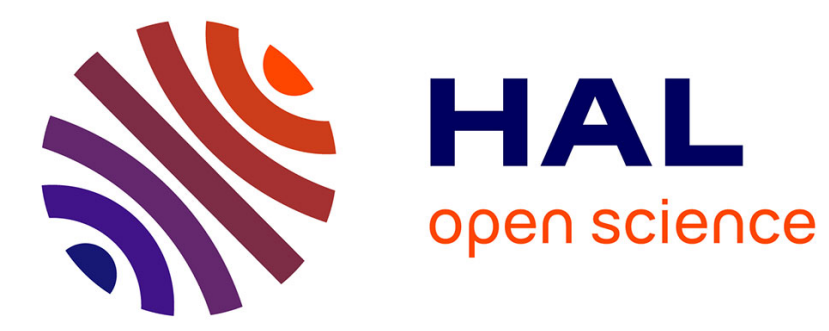

\title{
Common Rate Maximization in Cooperative Multiple Access Channels
}

\author{
Paul Ferrand, Jean-Marie Gorce, Claire Goursaud
}

\section{To cite this version:}

Paul Ferrand, Jean-Marie Gorce, Claire Goursaud. Common Rate Maximization in Cooperative Multiple Access Channels. [Research Report] 2012. hal-00742234

HAL Id: hal-00742234

https://hal.inria.fr/hal-00742234

Submitted on 16 Oct 2012

HAL is a multi-disciplinary open access archive for the deposit and dissemination of scientific research documents, whether they are published or not. The documents may come from teaching and research institutions in France or abroad, or from public or private research centers.
L'archive ouverte pluridisciplinaire HAL, est destinée au dépôt et à la diffusion de documents scientifiques de niveau recherche, publiés ou non, émanant des établissements d'enseignement et de recherche français ou étrangers, des laboratoires publics ou privés. 


\title{
Common Rate Maximization in Cooperative Multiple Access Channels
}

\author{
Paul Ferrand, Jean-Marie Gorce and Claire Goursaud \\ Université de Lyon, INRIA \\ INSA-Lyon, CITI-INRIA, F-69621, Villeurbanne, France \\ Email : paul.ferrand@insa-lyon.fr
}

\begin{abstract}
In this paper, we study the optimal power allocations in cooperative multiple access channels (CMACs), where we aim at maximizing the rate achievable by both sources simultaneously rather than the sum of achievable rates. Separating our study between the coherent and non-coherent case, we obtain closedform expressions for the optimal power allocations w.r.t. the outer bounds of the capacity region, as well as decode-and-forward and non-cooperative inner bounds. We point out during our resolution that the general CMAC model behaves as a multiple access relay channel (MARC), where a "virtual" relay node is introduced to represent the cooperation between the sources. This equivalent model simplifies the original power allocation problem. We finally show that the general cut-set outer bound on the capacity region of the equivalent MARC matches exactly the tightest known outer bound on the capacity region of the original CMAC.
\end{abstract}

\section{INTRODUCTION}

The study of the non-cooperative multiple access channel (MAC) traces back to Shannon in 1961. We refer the reader to $[1, \mathrm{Ch} .4]$ for a survey on the bibliography of the MAC. The cooperative case of the MAC, where sender nodes can communicate with one another, can be linked to studies on the MAC with generalized feedback as studied by Carleial [2], where the feedback signals are considered as direct transmission between the sources. In [3], Cover and Leung developped an inner decode-and-forward bound on the capacity of the MAC with feedback, which has been adapted by Laneman to the CMAC in [4]. An alternative model for a CMAC has been introduced by Willems [5] where senders are linked by a bitpipe of fixed capacity. In his paper, Willems characterized the capacity region in the discrete memoryless case, whereas an alternative proof for the Gaussian case has been presented by Bross et al. [6]. More recently, Tandon and Ulukus tightened the traditional cut-set outer bound on the capacity region of the CMAC in [7], using a dependence-balance relation between the source distributions. The dependence-balance has been introduced by Hekstra and Willems in [8] in the context of two-way channels.

Power allocation in the CMAC under an average power constraint at each node has been studied in [9] through a specific partial decode-and-forward implementation. A more general power allocation scheme has been derived in [10], still under a per-node power constraint. In [11], the authors studied the sum-rate maximization under a per-node power constraint and a fading channel hypothesis, where source nodes have access to the channel state information at all times. They derived the analytical form of the optimal solution and an efficient algorithm based on subgradient methods.

The paper is organized as follow. We consider various scheme on the CMAC model as described by Laneman [4]. We aim at maximizing the common rate achievable by both sources simultaneously. The first part of the paper treats the non-coherent case, under the hypothese that the source nodes cannot synchronize their transmission precisely enough to create a coherent addition of their signals at the destination. We derive optimal power allocations w.r.t. the common achievable rate for the cut-set outer bound as well as the non-cooperative MAC. We then describe a non-coherent decode and forward inner bound and the derivation of the optimal power allocation. In the second part of the paper we focus on the coherent case, extracting the optimal power allocation for the Laneman decode-and-forward inner bound [4]. We point out that in this case, the CMAC model behaves as an equivalent multiple access relay channel (MARC, see [12]), by introducing a "virtual" relay node concentrating the cooperative part of the transmission by the sources. We show that the outer bound on the capacity of this equivalent channel matches the tightest outer bound known to date on the capacity of the CMAC [7].

\section{MODEL DESCRIPTION}

\section{A. Network and channel model}

The cooperative multiple access channel (CMAC) is a network model composed of two sources nodes and a destination node, sometimes referred to as multiple access channel with user cooperation. In this paper, we consider a full-duplex CMAC, where nodes are able to send and receive information at the same time. Each source aims at transmitting its own message, possibly helping the other source along the way. We write $X_{i}$ and $Y_{i}$ the signals respectively sent and received by the sources $i \in\{1,2\}$, while the signal received by the destination is referred to as $Y_{D}$.

From [1, Th.18.4] we can write the upper bound on the capacity of this channel as the capacity of every cut in the network. Considering the transmission rates of sources 1 and 2 as $R_{1}$ and $R_{2}$ respectively, we write :

$$
\begin{aligned}
R_{1} & \leq I\left(X_{1} ; Y_{2}, Y_{D} \mid X_{2}\right) \\
R_{2} & \leq I\left(X_{2} ; Y_{1}, Y_{D} \mid X_{1}\right) \\
R_{1}+R_{2} & \leq I\left(X_{1}, X_{2} ; Y_{D}\right)
\end{aligned}
$$




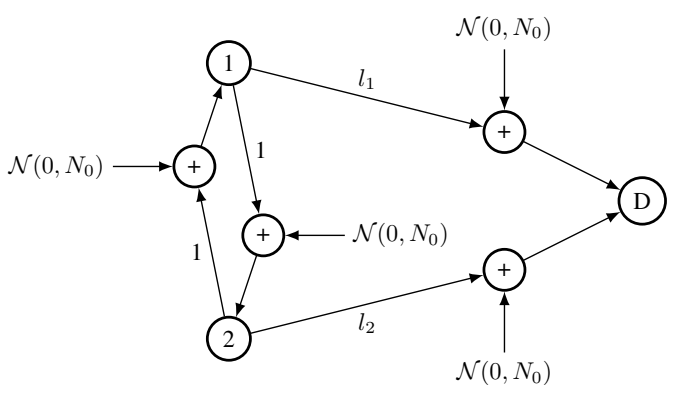

Fig. 1: Cooperative multiple access channel under a Gaussian hypothesis, where each node receive signals corrupted by white gaussian noise.

We focus in this paper on an analysis of the Gaussian CMAC (Fig.1). We consider that the each node is subject to a mean power constraint $P_{i}$, and that the sum of their transmission powers is such that $P_{1}+P_{2}=P_{\text {tot }}$. The signals between the nodes are scaled by a fixed coefficient $h_{i, j}$ and corrupted by white noise of density $N_{0}$. The signals from the source nodes to the destination have a potential correlation factor. We consider natural logarithms and our capacity results are thus in nats/s. In order to simplify the expression, we decide to normalize the power further by the value of the channel coefficient of the inter-source link $h_{1,2}$, e.g. changing $P_{i}$ into $\bar{P}_{i}=\left|h_{1,2}\right|^{2} P_{i}$. This leads us not to consider the 1-D link and 2-D link channel coefficients directly, but rather their relative quality w.r.t. to the inter-source link. We will write $l_{1}=h_{1, D}^{2} / h_{1,2}^{2}$ and $l_{2}=h_{2, D}^{2} / h_{1,2}^{2}$ in the remainder of the paper. Using these hypotheses, the derivation of the upper bound on the capacity of the CMAC can be written:

$$
\begin{aligned}
R_{1} & \leq \log \left(1+\left(1+l_{1}\right)\left(1-\rho^{2}\right) \bar{P}_{1}\right) \\
R_{2} & \leq \log \left(1+\left(1+l_{2}\right)\left(1-\rho^{2}\right) \bar{P}_{2}\right) \\
R_{1}+R_{2} & \leq \log \left(1+l_{1} \bar{P}_{1}+l_{2} \bar{P}_{2}+2 \rho \sqrt{l_{1} l_{2} \bar{P}_{1} \bar{P}_{2}}\right)
\end{aligned}
$$

We can note that the signals from the source nodes to the destination have a potential correlation factor $\rho$. This correlation stems from their cooperation, and requires a coherent transmission between both sources on top of a joint codebook design for the cooperative phase.

\section{B. Common rate}

Usually, there are tradeoffs to be made between the rates of different nodes in a multi-terminal network. As such, unlike the single link case, there are no unilateraly best achievable region in a multi-source channel model. We consider applications in small-scale networks where source nodes have constraints on their effective rates. Equations (2) define an upper bound on the region for achievable rates for the CMAC, but what we are actually interested in is which rate is achievable by both nodes. Going towards this description is straightforward. The common rate semi-line $R_{1}=R_{2}=R$ will intersect the convex closure of every possible rate regions obtained using (2) at a single point in realistic cases, allowing us to go from treating a region of achievable rates to a single rate variable $R$. This model is actually readily expanded into different relative demands on the rates of each node. For example, we may well transform the region $\left\{R_{1} \leq \ldots, R_{2} \leq \ldots, R_{1}+R_{2} \leq \ldots\right\}$ in (2) into the region $\{R \leq \ldots, \alpha R \leq \ldots,(1+\alpha) R \leq \ldots\}$ for some fixed value of $\alpha>0$. We would then obtain, as a result of our optimization problems, the value of $R$ at the intersection of the convex closure of possible rate regions with the semi-line $R_{1}=\alpha R_{2}$.

\section{NON-COHERENT CMAC}

Coherency is difficult to achieve in practical systems, due to the technological requirements. Both source have to send signals in a synchronous manner in order to achieve a beamforming gain at the destination. They also have to share a common codebook and choose their symbols cooperatively so that their respective signals do add at the receiver.

\section{A. Simple bounds}

When either of the coherency technical requirements aren't met, the upper bound reduces to a simpler case with $\rho=0$. In that case, under the common rate framework, we can obtain the optimal power allocation w.r.t. to the upper bound by solving a convex optimization problem. We have the following proposition:

Proposition 1. We consider a full-duplex CMAC as represented on Fig.1. If the sources are not able to signal coherently at the destination, the optimal power allocation for both sources to attain the maximum common rate $R_{\mathrm{CMAC}}^{+}$with respect to the upper bound is:

$\bar{P}_{1}= \begin{cases}\bar{P}_{\mathrm{tot}}-\frac{\sqrt{\left(2+l_{1}+l_{2}\right)^{2}+4 l_{1}\left(1+l_{2}\right)^{2} \bar{P}_{\mathrm{tot}}}-\left(2+l_{1}+l_{2}\right)}{2\left(1+l_{2}\right)^{2}} & \text { if } l_{1}>l_{2} \\ \frac{\bar{P}_{\mathrm{tot}}}{2} & \text { if } l_{1}=l_{2} \\ \frac{\sqrt{\left(2+l_{1}+l_{2}\right)^{2}+4 l_{2}\left(1+l_{1}\right)^{2} \bar{P}_{\mathrm{tot}}}-\left(2+l_{1}+l_{2}\right)}{2\left(1+l_{1}\right)^{2}} & \text { if } l_{1}<l_{2}\end{cases}$

The value of $R_{\mathrm{CMAC}}^{+}$is obtained by plugging these allocations in the expressions in (2), along with $\rho=0$.

Proof: We write the optimization problem as:

$$
\begin{array}{cll}
\underset{R, \bar{P}_{1}, \bar{P}_{2}}{\operatorname{minimize}} & -R & \\
\text { subject to } & R & \leq \log \left(1+\left(1+l_{1}\right) \bar{P}_{1}\right) \\
& R & \leq \log \left(1+\left(1+l_{2}\right) \bar{P}_{2}\right) \\
& 2 R & \leq \log \left(1+l_{1} \bar{P}_{1}+l_{2} \bar{P}_{2}\right)
\end{array}
$$

We write the lagrangian function $\mathcal{L}\left(\lambda_{1}, \lambda_{2}, \lambda_{3}, \mu, R, \bar{P}_{1}, \bar{P}_{2}\right)$ as follow [13]:

$$
\begin{aligned}
\mathcal{L}=-R & +\lambda_{1}\left[R-\log \left(1+\left(1+l_{1}\right) \bar{P}_{1}\right)\right] \\
& +\lambda_{2}\left[R-\log \left(1+\left(1+l_{2}\right) \bar{P}_{2}\right)\right] \\
& +\lambda_{3}\left[2 R-\log \left(1+l_{1} \bar{P}_{1}+l_{2} \bar{P}_{2}\right)\right] \\
& +\mu\left[\bar{P}_{1}+\bar{P}_{2}-\bar{P}_{\text {tot }}\right]
\end{aligned}
$$


The partial derivatives of the lagrangian function are thus:

$$
\begin{aligned}
& \frac{\partial \mathcal{L}}{\partial R}=\lambda_{1}+\lambda_{2}+2 \lambda_{3}-1 \\
& \frac{\partial \mathcal{L}}{\partial \bar{P}_{1}}=-\frac{\lambda_{1}\left(1+l_{1}\right)}{1+\left(1+l_{1}\right) \bar{P}_{1}}-\frac{\lambda_{3} l_{1}}{1+l_{1} \bar{P}_{1}+l_{2} \bar{P}_{2}}+\mu \\
& \frac{\partial \mathcal{L}}{\partial \bar{P}_{1}}=-\frac{\lambda_{2}\left(1+l_{2}\right)}{1+\left(1+l_{2}\right) \bar{P}_{2}}-\frac{\lambda_{3} l_{2}}{1+l_{1} \bar{P}_{1}+l_{2} \bar{P}_{2}}+\mu
\end{aligned}
$$

The Karush-Kuhn-Tucker conditions tell us that either the constraint inequalities are met with equality, or the associated $\lambda_{i}$ is equal to zero [13]. Furthermore, we have $\mu \neq 0$. We can proceed by elimination:

- Having only $\lambda_{1} \neq 0$ or $\lambda_{2} \neq 0$ leads to a violation of the condition $\mu \neq 0$.

- For $\lambda_{3} \neq 0$ and $\lambda_{1}=0, \lambda_{2}=0$ we have that necessarily $l_{1}=l_{2}$ and in that case $\bar{P}_{1}=\bar{P}_{2}=\bar{P}_{\text {tot }} / 2$.

- Having $\lambda_{1} \neq 0, \lambda_{2} \neq 0$ and $\lambda_{3}$ arbitrary is impossible, since the sum of the first two inequalities in (4) is stricly higher that the third constraint for any $P_{1}, P_{2} \neq 0$.

We must thus study the cases where the $\left(\lambda_{1} \neq 0, \lambda_{3} \neq 0, \lambda_{2}=\right.$ $0)$ and $\left(\lambda_{2} \neq 0, \lambda_{3} \neq 0, \lambda_{1}=0\right)$. We detail the case where $\lambda_{2}=0$, the proof in the second case being similar. We are left with the following relations from the partial derivatives:

$$
\begin{aligned}
\mu & =\frac{\lambda_{3} l_{2}}{1+l_{1} \bar{P}_{1}+l_{2} \bar{P}_{2}} \\
\frac{\lambda_{3}\left(l_{2}-l_{1}\right)}{1+l_{1} \bar{P}_{1}+l_{2} \bar{P}_{2}} & =\frac{\left(1+l_{1}\right) \lambda_{1}}{1+\left(1+l_{1}\right) \bar{P}_{1}}
\end{aligned}
$$

From $\mu \neq 0$ and $\lambda_{1} \geq 0$, we can deduce from these two equations that we need to have $l_{2}>l_{1}, \lambda_{3} \neq 0$ and $\lambda_{1} \neq 0$ in order to have $\lambda_{2}=0$. In this situation, we have the first and last inequation constraints from (4) met with equality. Through basic algebra, we can conclude that the optimal value for $\bar{P}_{1}$ is a root of a degree two polynomial:

$$
\left(1+l_{1}\right)^{2} \bar{P}_{1}^{2}+\left(2+l_{1}+l_{2}\right) \bar{P}_{1}-l_{2} \bar{P}_{\text {tot }}=0
$$

This polynomial has a negative and a positive root. Since we can't have negative powers, the optimal solution for our problem is the positive root as written in the proposition. At last, setting $\lambda_{1}=0$ leads to a similar derivation for $\bar{P}_{2}$, and by noting that $\bar{P}_{1}=\bar{P}_{\text {tot }}-\bar{P}_{2}$ we complete the expressions in (3) and the proof.

In this network model, an obvious lower bound for the capacity region is the simple multiple access channel, where nodes do not cooperate with each other. In that case, the capacity region is written as [1, Ch.4]:

$$
\begin{aligned}
R_{1} & \leq \log \left(1+l_{1} \bar{P}_{1}\right) \\
R_{2} & \leq \log \left(1+l_{2} \bar{P}_{2}\right) \\
R_{1}+R_{2} & \leq \log \left(1+l_{1} \bar{P}_{1}+l_{2} \bar{P}_{2}\right)
\end{aligned}
$$

We can see that this region is very close to (2), and we can use the same method as Prop. 1 to obtain a similar result for the non-cooperative MAC:
Proposition 2. We consider a MAC, where nodes do not send information to each other. The optimal power allocation for both sources to attain the maximum common rate $R_{\mathrm{MAC}}^{-}$is:

$$
\bar{P}_{1}= \begin{cases}\bar{P}_{\mathrm{tot}}-\frac{\sqrt{\left(l_{1}+l_{2}\right)^{2}+4 l_{1} l_{2}^{2} \bar{P}_{\mathrm{tot}}}-\left(l_{1}+l_{2}\right)}{2 l_{2}^{2}} & \text { if } l_{1}>l_{2} \\ \frac{\bar{P}_{\mathrm{tot}}}{2} & \text { if } l_{1}=l_{2} \\ \frac{\sqrt{\left(l_{1}+l_{2}\right)^{2}+4 l_{2} l_{1}^{2} \bar{P}_{\mathrm{tot}}}-\left(l_{1}+l_{2}\right)}{2 l_{1}^{2}} & \text { if } l_{1}<l_{2}\end{cases}
$$

The value of $R_{\mathrm{MAC}}^{-}$is obtained by plugging these allocations in the expressions in (10).

Proof: The proof follows from Prop.1, replacing the terms $\left(1+l_{i}\right)$ by $l_{i}$ in the optimization problem (4).

\section{B. Decode-and-forward lower bound}

In his thesis [4], Laneman provides a decode-and-forward lower bound for the CMAC based on the Cover-Leung inner bound for the MAC with feedback [3]. This lower bound requires coherent cooperation between the sources and as such is not adapted to the non-coherent CMAC. In this section, we develop a non-coherent decode-and-forward lower bound and its associated power allocation. We have the following proposition:

Proposition 3. The achievable rates for decode-and-forward transmission over a non-coherent CMAC is given by the region of rates $\left(R_{1}, R_{2}\right)$ satisfying:

$$
\begin{aligned}
R_{1} & \leq I\left(X_{1} ; Y_{2} \mid U_{1}\right) \\
R_{2} & \leq I\left(X_{2} ; Y_{1} \mid U_{2}\right) \\
R_{1}+R_{2} & \leq I\left(X_{1}, X_{2} ; Y_{D}\right)
\end{aligned}
$$

for some distribution $p\left(u_{1}\right) p\left(u_{2}\right) p\left(x_{1} \mid u_{1}\right) p\left(x_{2} \mid u_{2}\right)$ on the support set of $\left(U_{1}, U_{2}, X_{1}, X_{2}\right)$.

Proof: Laneman's result [4] gives the decode-and-forward region for a coherent CMAC as follow:

$$
\begin{aligned}
& R_{1} \leq I\left(X_{1} ; Y_{2} \mid U\right) \\
& R_{2} \leq I\left(X_{2} ; Y_{1} \mid U\right) \\
& R_{1}+R_{2} \leq I\left(X_{1}, X_{2} ; Y_{D}\right)
\end{aligned}
$$

for some distribution $p(u) p\left(x_{1}, x_{2} \mid u\right)$. Since we only consider the non-coherent case, we have to generate the codewords separately. We thus split the random variable $U$ into the random vector $\left(U_{1}, U_{2}\right)$ such that $U_{1}$ is independent of $U_{2}$. Furthermore, setting the conditional random variable $X_{1} \mid U_{1}$ independent of $U_{2}$ and $X_{2} \mid U_{2}$ independent of $U_{1}$ leads to the region in the proposition. It is possible to prove the same result through irregular block-Markov encoding with binning at the sources and forward decoding, although constrained by the paper length we cannot detail this method.

We write $X_{1}=\sqrt{P_{1,1}} V_{1}+\sqrt{P_{1,2}} U_{1}$ and $X_{2}=\sqrt{P_{2,1}} V_{2}+$ $\sqrt{\bar{P}_{2,2}} U_{2}$, with $\left(U_{1}, U_{2}, V_{1}, V_{2}\right)$ being i.i.d. normal random variables of mean 0 and variance 1 and $\bar{P}_{1,1}+\bar{P}_{1,2}+\bar{P}_{2,1}+$ 
$\bar{P}_{2,2}=\bar{P}_{\text {tot }}$. The region thus reduces as follow for the gaussian CMAC case:

$$
\begin{aligned}
R_{1} & \leq \log \left(1+\bar{P}_{1,1}\right) \\
R_{2} & \leq \log \left(1+\bar{P}_{2,1}\right) \\
R_{1}+R_{2} & \leq \log \left(1+l_{1}\left(\bar{P}_{1,1}+\bar{P}_{1,2}\right)+l_{2}\left(\bar{P}_{2,1}+\bar{P}_{2,2}\right)\right)
\end{aligned}
$$

Once again, the form of the region is similar to (2) and (10), and we can derive the optimal power allocation for the common rate case in the fashion of Prop. 1 and 2:

Proposition 4. We consider a full-duplex CMAC as represented on Fig. I. If the sources are not able to signal coherently at the destination, the optimal power allocation for both sources to attain the maximum common rate $R_{\mathrm{CMAC}}^{-}$with respect to the decode-and-forward lower bound is as follow:

- If $4\left(\frac{l_{1}+l_{2}}{2}-1\right) \geq P_{\text {tot }}$, then $\bar{P}_{1,2}=\bar{P}_{2,2}=0$ and $\bar{P}_{1,1}=$ $\bar{P}_{2,1}=\bar{P}_{\text {tot }} / 2$. Otherwise,

- If $l_{1}=l_{2}$, then $\bar{P}_{1,1}=\bar{P}_{2,1}$ and $\bar{P}_{1,2}=\bar{P}_{2,2}=\bar{P}_{\text {tot }} / 2-$ $\bar{P}_{1,1}$ where:

$$
\bar{P}_{1,1}=\min \left\{\sqrt{1+l_{1} P_{\mathrm{tot}}}-1, \frac{\bar{P}_{\mathrm{tot}}}{2}\right\}
$$

- If $l_{1}<l_{2}$, we have $\bar{P}_{1,2}=0, \bar{P}_{2,2}=\max \left\{\bar{P}_{\text {tot }}-\right.$ $\left.2 \bar{P}_{2,1}, 0\right\}$ and :

$\bar{P}_{1,1}=\bar{P}_{2,1}=\min \left\{\frac{\sqrt{\left(2+l_{2}-l_{1}\right)^{2}+4 l_{2} \bar{P}_{\mathrm{tot}}}-\left(2+l_{2}-l_{1}\right)}{2}, \frac{P_{\mathrm{tot}}}{2}\right\}$

- If $l_{1}>l_{2}$, we have $\bar{P}_{2,2}=0, \bar{P}_{1,2}=\max \left\{\bar{P}_{\text {tot }}-\right.$ $\left.2 \bar{P}_{1,1}, 0\right\}$ and :

$\bar{P}_{1,1}=\bar{P}_{2,1}=\min \left\{\frac{\sqrt{\left(2+l_{1}-l_{2}\right)^{2}+4 l_{1} \bar{P}_{\text {tot }}}-\left(2+l_{1}-l_{2}\right)}{2}, \frac{P_{\text {tot }}}{2}\right\}$

The value of $R_{\mathrm{CMAC}}^{-}$is obtained by plugging these allocations in the expressions in (15).

Remark. - We can analyze from the last two cases of the proposition that when $l_{1}>l_{2}$ (respectively $l_{2}>l_{1}$ ), node 2 doesn't relay any information at all since node 1 has a better direct path to the destination. The non-coherent decode-andforward thus act as the superposition of a direct link and a relay channel.

Proof: We proceed in the same manner as Prop.1. The partial derivatives of the Lagrangian function $\mathcal{L}$ for the optimization problem are:

$$
\begin{aligned}
\frac{\partial \mathcal{L}}{\partial R} & =\lambda_{1}+\lambda_{2}+2 \lambda_{3}-1 \\
\frac{\partial \mathcal{L}}{\partial \bar{P}_{1,1}} & =-\frac{\lambda_{1}}{1+\bar{P}_{1,1}}-\frac{\lambda_{3} l_{1}}{A}+\mu \\
\frac{\partial \mathcal{L}}{\partial \bar{P}_{1,2}} & =-\frac{\lambda_{3} l_{1}}{A}+\mu \\
\frac{\partial \mathcal{L}}{\partial \bar{P}_{2,1}} & =-\frac{\lambda_{2}}{1+\bar{P}_{2,1}}-\frac{\lambda_{3} l_{2}}{A}+\mu \\
\frac{\partial \mathcal{L}}{\partial \bar{P}_{2,2}} & =-\frac{\lambda_{3} l_{2}}{A}+\mu
\end{aligned}
$$

where $A=1+l_{1}\left(\bar{P}_{1,1}+\bar{P}_{1,2}\right)+l_{2}\left(\bar{P}_{2,1}+\bar{P}_{2,2}\right)$. Setting $\lambda_{3}=0$ directly leads to the conclusion that $\bar{P}_{1,2}=\bar{P}_{2,2}=0$ and $\bar{P}_{1,1}=\bar{P}_{2,1}=\bar{P}_{t o t} / 2$. Furthermore, since both constraints on $R$ are active but not the one on $2 R$, we have the condition that $(1+\bar{P} \text { tot } / 2)^{2} \leq 1+\left(l_{1}+l_{2}\right) \bar{P}_{\text {tot }} / 2$. After simplifications, it leads to the first bullet of the proposition.

For $\lambda_{3} \neq 0$, we can directly deduce that $\lambda_{1}=\lambda_{2}=0$ and $l_{1}=l_{2}$ from the partial derivatives equations. We thus have $2 R=\log \left(1+l_{1} \bar{P}_{\text {tot }}\right)$. The power allocation at this point is almost arbitrary, but in order to respect every constraint, we need $R \leq \log \left(1+\bar{P}_{1,1}\right)$ and $R \leq \log \left(1+\bar{P}_{2,1}\right)$. We can set $\bar{P}_{1,1}=\overline{\bar{P}}_{2,1}$ and $\bar{P}_{1,2}=\bar{P}_{2,2}$ and thus we have the condition in the second bullet.

For the last cases, we need to suppose one of the $\bar{P}_{1,2}$, $\bar{P}_{2,2}$ is equal to 0 . We illustrate the case $\bar{P}_{2,2}=0$. We have that $\lambda_{3} \neq 0$, thus implying $\lambda_{1}=0$ and from $\lambda_{2} \geq 0$ we can deduce $l_{1}>l_{2}$ and $\lambda_{2} \neq 0$. The second and third inequality constraints are thus active. We thus solve $\left(1+\bar{P}_{2,1}\right)^{2}=1+l_{2} \bar{P}_{2,1}+l_{1}\left(\bar{P}_{\text {tot }}-\bar{P}_{2,1}\right)$ for $\bar{P}_{2,1}$. In the end, we need to have $\bar{P}_{1,1} \geq \bar{P}_{2,1}$ to respect the first inequality constraint. The derivation is similar for $\bar{P}_{1,2}=0$.

\section{COHEREnT CMAC}

In cooperative networks, the source terminals are by design able to communicate with each other and thus construct a joint codebook. If they can achieve the necessary synchronization to produce a beamformed signal at the destination terminals the potential gain from cooperation is even greater than the non-coherent case.

\section{A. Coherent decode-and-forward lower bound}

The Laneman bound [4] for the coherent CMAC writes:

$$
\begin{aligned}
R_{1} \leq & \log \left(1+\bar{P}_{1,1}\right) \\
R_{2} \leq & \log \left(1+\bar{P}_{2,1}\right) \\
R_{1}+R_{2} \leq & \log \left(1+l_{1}\left(\bar{P}_{1,1}+\bar{P}_{1,2}\right)+l_{2}\left(\bar{P}_{2,1}+\bar{P}_{2,2}\right)\right. \\
& \left.+2 \sqrt{l_{1} l_{2} \bar{P}_{1,2} \bar{P}_{2,2}}\right)
\end{aligned}
$$

The gain comes from the coherent combination of signals in the sum-rate, the decode and forward transmission still being limited by the information both sources are able to exchange with each other as seen in the first two inequations in (16). We can see from the sum-rate constraint that the power is split at each node ; a part $\bar{P}_{i}^{1}$ of the nodes' power will be used to transmit their own information, while another part $P_{i}^{2}$ will be used for the cooperation and will add coherently in the sumrate. We note that we can rewrite the right-hand side of the last inequation as:

$$
\log \left(1+l_{1} \bar{P}_{1,1}+l_{2} \bar{P}_{2,1}+\left(\sqrt{l_{1} \bar{P}_{1,2}}+\sqrt{l_{2} \bar{P}_{2,2}}\right)^{2}\right)
$$

The coherent parts of the signal thus take the form of a Multiple Input-Single Output (MISO) channel from a "virtual" 
source symbolizing the cooperation between the two transmitters. Under a total power constraint, we can simplify the problem by allocating some power $\bar{P}_{\text {eq }}$ to the cooperative part. For any $\bar{P}_{\text {eq }}$ and channel coefficients $\left(l_{1}, l_{2}\right)$, we know the optimal power allocation for the MISO channel as [14]:

$$
\bar{P}_{1,2}=\frac{l_{1}}{l_{1}+l_{2}} \bar{P}_{\mathrm{eq}} \quad \bar{P}_{2,2}=\frac{l_{2}}{l_{1}+l_{2}} \bar{P}_{\mathrm{eq}}
$$

Injecting (18) into (16) gives the following region, with $\hat{l}=$ $\left(l_{1}+l_{2}\right)$ :

$$
\begin{aligned}
R_{1} & \leq \log \left(1+\bar{P}_{1,1}\right) \\
R_{2} & \leq \log \left(1+\bar{P}_{2,1}\right) \\
R_{1}+R_{2} & \leq \log \left(1+l_{1} \bar{P}_{1,1}+l_{2} \bar{P}_{2,1}+\hat{l} \bar{P}_{\mathrm{eq}}\right)
\end{aligned}
$$

Using this rate region, we can derive the optimal power allocation maximizing the common achievable rate in the coherent decode-and-forward case:

Proposition 5. We consider a full-duplex CMAC as represented on Fig.1. If the sources are able to signal coherently at the destination, the optimal power allocation for both sources to attain the maximum common rate $R_{\mathrm{CMAC}, \mathrm{coh}}^{-}$with respect to the coherent decode-and-forward lower bound is as follow:

$$
\begin{aligned}
\bar{P}_{1,1} & =\min \left\{\frac{\sqrt{(2+\hat{l})^{2}+4 \hat{l} \bar{P}_{\mathrm{tot}}}-(2+\hat{l})}{2}, \frac{\bar{P}_{\mathrm{tot}}}{2}\right\} \\
\bar{P}_{2,1} & =\bar{P}_{1,1} \\
\bar{P}_{\mathrm{eq}} & =\max \left\{\bar{P}_{\mathrm{tot}}-2 \bar{P}_{1,1}, 0\right\}
\end{aligned}
$$

The original power $\bar{P}_{1,2}$ and $P_{2,2}$ are computed through (18) and the value of $R_{\mathrm{CMAC}, \mathrm{coh}}^{-}$is obtained by plugging these allocations in the expressions in (19).

Proof: We only outline the proof, which follows the ones from the previous propositions. Writing the Lagrangian function and its partial derivatives w.r.t. the power variables $\bar{P}_{1,1}, \bar{P}_{2,1}$ and $\bar{P}_{\text {eq }}$ we show that every rate constraint is met with equality. As a result, we have that $\bar{P}_{1,1}=\bar{P}_{2,1}$. Noting that $\bar{P}_{\text {eq }}=\bar{P}_{\text {tot }}-2 \bar{P}_{1,1}$, we solve a second degree polynomial on $\bar{P}_{1,1}$ to obtain the result in (20). We complete the proof by ensuring the intrinsic bounds on the power variables are met in every case.

\section{B. Coherent upper-bound with the virtual source}

The insight from the decode-and-forward lower bound in the previous section is that the nodes split their informations into two uncorrelated parts, where some power is set aside for coherent cooperation with each other. This cooperation takes the form of a MISO channel. The network is thus somehow equivalent to a multiple access relay channel (MARC) [12] as represented on Fig.2. We argue in this section that the "virtual" node representation leads to a tighter upper bound on the capacity of the CMAC, as well as an intuitive representation of the cooperation limits for this network.

In the equivalent channel, the source nodes do not receive information, and signals towards a source are thus sent to

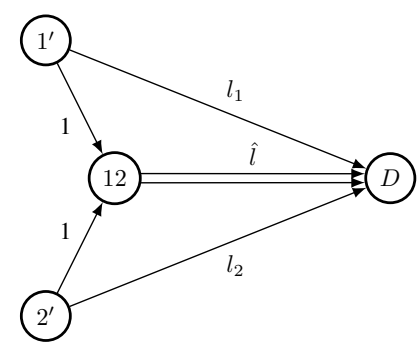

Fig. 2: Equivalent MARC model for the cooperative multiple access channel. The "virtual node" 12 represents the coherent cooperation between nodes 1 and 2 , over the equivalent channel $\hat{l}=l_{1}+l_{2}$.

the cooperation node. Each source splits its information, and following the notation of the original CMAC model, we thus have $X_{1}=\left(X_{1,1}, X_{1,2}\right)$ and $X_{2}=\left(X_{2,1}, X_{2,2}\right)$. On the equivalent MARC model (Fig.2), source $1^{\prime}$ will send information through $X_{1,1}$ and $2^{\prime}$ through $X_{2,1}$, while the virtual node 12 will use $X_{\text {eq }}=\left(X_{1,2}, X_{2,2}\right)$ as its emission variable, and $Y_{\mathrm{eq}}=\left(Y_{1}, Y_{2}\right)$ as its reception variable. In the cutset upper bound, Gaussian random variable at the emitter maximized the entropy. By design of the equivalent network, $X_{i, 1}$ and $X_{i, 2}$ are independent of each other, while $X_{1,2}$ and $X_{2,2}$ have a correlation coefficient of 1 , which means that they are deterministic linear functions of one another. We thus have $X_{1,2} \leftrightarrow X_{2,2} \leftrightarrow\left(Y_{1}, Y_{D}\right)$ and $X_{2,2} \leftrightarrow X_{1,2} \leftrightarrow\left(Y_{2}, Y_{D}\right)$ as Markov chains in the equivalent channel. The equivalent MARC channel is also non-coherent, as the sources and relay distribution are independent of one another.

Applying the cutset upper bound from [1, Th.18.4] to the equivalent MARC model gives the following region:

$$
\begin{aligned}
R_{1} & \leq I\left(X_{1,1} ; Y_{2}, Y_{D} \mid X_{\mathrm{eq}}, X_{2,1}\right) \\
R_{2} & \leq I\left(X_{2,1} ; Y_{1}, Y_{D} \mid X_{\mathrm{eq}}, X_{1,1}\right) \\
R_{1}+R_{2} & \leq I\left(X_{1,1}, X_{2,1}, X_{\mathrm{eq}} ; Y_{D}\right) \\
R_{1} & \leq I\left(X_{1,1}, X_{\mathrm{eq}} ; Y_{D} \mid X_{2,1}\right) \\
R_{2} & \leq I\left(X_{2,1}, X_{\mathrm{eq}} ; Y_{D} \mid X_{1,1}\right) \\
R_{1}+R_{2} & \leq I\left(X_{1,1}, X_{2,1} ; Y_{\mathrm{eq}}, Y_{D} \mid X_{\mathrm{eq}}\right)
\end{aligned}
$$

The rate constraint (23a) can be matched to the original cutset upper bound from (1) as follow:

$$
\begin{aligned}
I\left(X_{1} ; Y_{2}, Y_{D} \mid X_{2}\right) & =I\left(X_{1,1}, X_{1,2} ; Y_{2}, Y_{D} \mid X_{2,1}, X_{2,2}\right) \\
& =I\left(X_{1,1} ; Y_{2}, Y_{D} \mid X_{2,1}, X_{2,2}, X_{1,2}\right) \\
& +I\left(X_{1,2} ; Y_{2}, Y_{D} \mid X_{2,1}, X_{2,2}\right) \\
& =I\left(X_{1,1} ; Y_{2}, Y_{D} \mid X_{2,1}, X_{\mathrm{eq}}\right)
\end{aligned}
$$

The second equality follows from the chain rule on mutual information, and the second information term is equal to 0 because $X_{2,2} \leftrightarrow X_{1,2} \leftrightarrow\left(Y_{2}, Y_{D}\right)$. We proceed in the same manner for (23b) and (23c), and we can thus conclude that the region (23) is contained in the region (1). The remaining inequations in (23) bound more precisely the capacity region of the CMAC. Inequations (23d) and (23e) represent an upper 


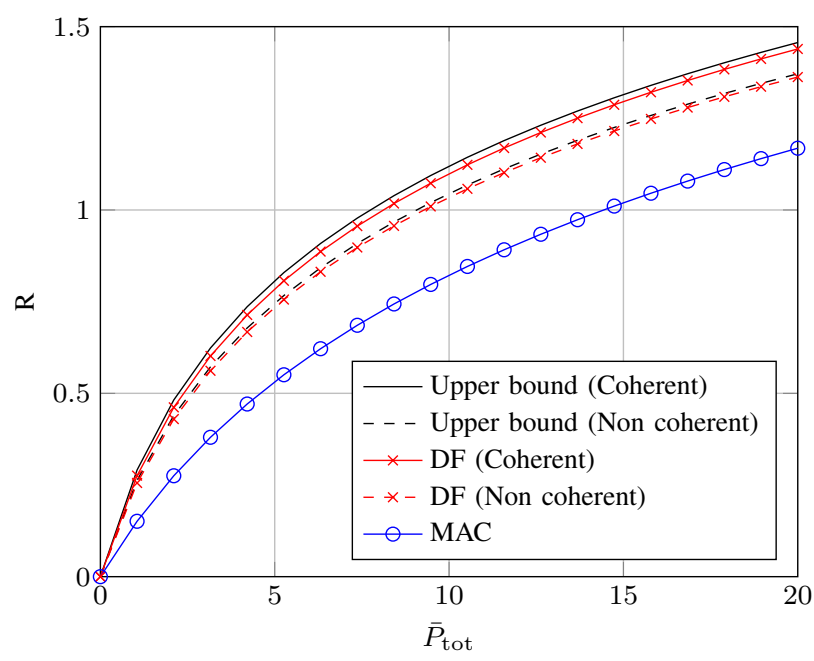

Fig. 3: Upper bounds and decode-and-forward lower bounds on the common achievable rate in the coherent and noncoherent CMACs, with $l_{1}=0.8$ and $l_{2}=0.2$.

bound on the rate of each source if the cooperation is purely directed towards a specific source node, e.g. (23d) is the maximum rate source $1^{\prime}$ may attain when the virtual node 12 only transmits the former's information. The last inequation's meaning is of particular interest, since it represents the maximum rate both source may transmit to the cooperating node. If we write the Gaussian application of (23f), we obtain:

$$
\begin{aligned}
R_{1}+R_{2} & \leq \log \left(1+\left(1+l_{1}\right) \bar{P}_{1,1}+\left(1+l_{2}\right) \bar{P}_{2,1}\right. \\
& \left.+\left(1+l_{1}+l_{2}\right) \bar{P}_{1,1} \bar{P}_{1,2}\right)
\end{aligned}
$$

This expression matches the dependence-balance bound [8] and its application to CMAC channels in [7]. The dependencebalance bound stems from a limit between the correlation of source distributions, and leads to a tighter upper bound on the capacity of cooperative networks. While it is still unclear how to mathematically relate the results from [7] to the expressions in this paper, the equivalent channel model gives interesting insights on how to extract dependence-balance bounds from arbitrary networks through a simple application of the general cut-set bound.

We plot the rates obtained with the power allocations in this paper on Fig.3. We use an example where links to the destination are asymmetrical and worse than the inter-source link, a situation where we expect the most gains from source cooperation. The coherent upper-bound case uses the results on the equivalent MARC presented in this section rather than the classical cutset bound. While it is possible to extract an optimal power allocation involving the root of a degree 3 polynomial for this upper bound, it is not pratictal and we use a numerical optimization algorithm for this application. We can see that both the coherent and non-coherent cooperation provide great gains over the non-cooperating MAC, and that the decodeand-forward bounds are very close to the upper bounds.

\section{CONClusion}

In this paper, we studied power allocation in cooperative multiple access channels (CMACs), where we aimed at maximizing the common rate attainable by both sources. We considered both coherent and non-coherent network to differentiate between the cases where sources were not able to produce a beamformed signal at the destination. We obtained closed-form optimal power-allocations for the cutset upper bound and decode-and-forward lower bounds, as well as the non-cooperative multiple access channel. Through our studies, we identified an equivalency between CMACs and multiple access relay channels (MARCs), where sources transmit to the destination with the help of a relay. We proposed a method to match coherent CMACs to non-coherent MARCs in order to simplify the expressions of the cut-set outer bound. Through this transformation, we identified tighter constraints on the rate region, and we give in particular an intuitive meaning to the existing dependence-balance bound on CMACs from [7].

The perspectives of this work lie mainly into the extension of the equivalent representation of coherent networks. While the equivalent MARC's outer bound on the capacity region is included in the original CMAC capacity region, we lack a complete constructive proof of the equivalency. Once the theory is sound, we aim at extending this approach to more complex networks with additional sources, since this approach could greatly simplify the expression of capacity bounds on medium-sized networks.

\section{REFERENCES}

[1] Abbas El Gamal and Young-Han Kim, Network Information Theory, Cambridge University Press, Ed., 2011.

[2] A. Carleial, "Multiple-access channels with different generalized feedback signals," IEEE Transactions on Information Theory, vol. 28, no. 6, pp. 841-850, 1982.

[3] T. Cover and C. Leung, "An achievable rate region for the multipleaccess channel with feedback," IEEE Transactions on Information Theory, vol. 27, no. 3, pp. 292-298, 1981.

[4] J. N. Laneman, "Cooperative Diversity in Wireless Networks: Algorithms and Architectures," Ph.D. dissertation, Massachusetts Institute of Technology, 2002.

[5] F. Willems, "The discrete memoryless multiple access channel with partially cooperating encoders (Corresp.)," IEEE Transactions on Information Theory, vol. 29, no. 3, pp. 441-445, 1983.

[6] S. I. Bross, A. Lapidoth, and M. A. Wigger, "The Gaussian MAC with conferencing encoders," in Proc. IEEE Int. Symp. Information Theory ISIT 2008, 2008, pp. 2702-2706.

[7] R. Tandon and S. Ulukus, "Dependence Balance Based Outer Bounds for Gaussian Networks With Cooperation and Feedback," IEEE Transactions on Information Theory, vol. 57, no. 7, pp. 4063-4086, 2011.

[8] A. P. Hekstra and F. M. J. Willems, "Dependence balance bounds for single-output two-way channels," IEEE Transactions on Information Theory, vol. 35, no. 1, pp. 44-53, 1989.

[9] A. Sendonaris, E. Erkip, and B. Aazhang, "User cooperation diversity. Part I. System description," Communications, IEEE Transactions on, vol. 51, no. 11, pp. 1927 - 1938, nov. 2003.

[10] W. Mesbah and T. N. Davidson, "Optimized Power Allocation for Pairwise Cooperative Multiple Access," IEEE Transactions on Signal Processing, vol. 56, no. 7, pp. 2994-3008, 2008.

[11] O. Kaya and S. Ulukus, "Power control for fading cooperative multiple access channels," Wireless Communications, IEEE Transactions on, vol. 6, no. 8, pp. 2915-2923, 2007.

[12] G. Kramer and A. J. van Wijngaarden, "On the white Gaussian multipleaccess relay channel," in Proc. IEEE Int Information Theory Symp, 2000. 
[13] Stephen Boyd and Lieven Vanderberghe, Convex Optimization. Cambridge University Press, 2004.

[14] John G. Proakis and Masoud Salehi, Digital Communications, 5th ed. McGraw-Hill, 2008 